$1-1-1969$

\title{
Experiments to control the alfalfa weevil with low- volume and ultra-low-volume spray treatments
}

\author{
C. K. Dorsey \\ L. P. Stevens
}

Follow this and additional works at: https://researchrepository.wvu.edu/ wv_agricultural_and_forestry_experiment_station_bulletins

\section{Digital Commons Citation}

Dorsey, C. K. and Stevens, L. P., "Experiments to control the alfalfa weevil with low-volume and ultra-low-volume spray treatments" (1969). West Virginia Agricultural and Forestry Experiment Station Bulletins. 579T.

https://researchrepository.wvu.edu/wv_agricultural_and_forestry_experiment_station_bulletins/685 @ WVU. It has been accepted for inclusion in West Virginia Agricultural and Forestry Experiment Station Bulletins by an authorized administrator of The Research Repository @ WVU. For more information, please contact ian.harmon@mail.wvu.edu. 
Digitized by the Internet Archive in 2010 with funding from

Lyrasis Members and Sloan Foundation 
Experiments to Control the Alfalfa Weevil with Low-Volume and Ultra-Low-Volume Spray Treatments

BULLETIN 579T-MAY 1969

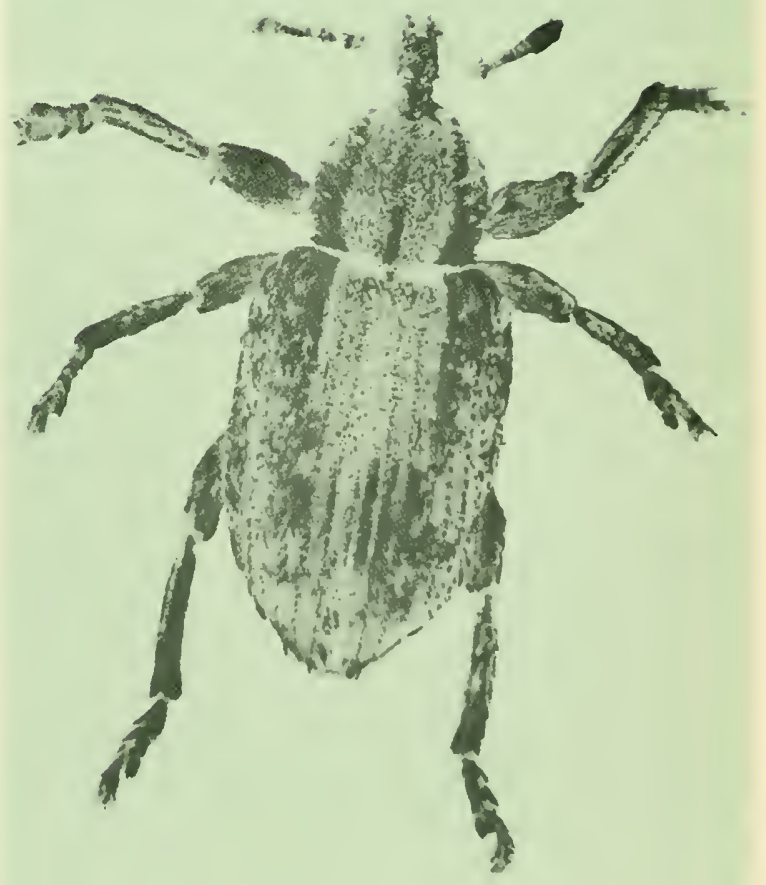




\section{The Authors}

C. K. Dorsey is Entomologist; L. P. Stevens is Assistant Horticulturist in charge of the Reedsville Experiment Farm.

\section{Acknowledgments}

The assistance of Dr. G. A. Jung, Agronomist, in procuring hay yield data is gratefully appreciated.

Marlow Pumps, Midland Park, N. J., furnished the Econo-Mist sprayer used in experiments in 1966.

WESt Virginia University

Agricultural Experiment Station

College of Agriculture and Forestry

A. H. Vanlandingham, Director

Morgantown 


\section{Experiments to Control the Alfalfa}

Weevil With Low-Volume and Ultra-

Low-Volume Spray Treatments

\section{K. DORSEY and L. P. STEVENS}

There has been increasing interest during the past five years in more effective ways of applying pesticides. New equipment and pesticide formulations have been developed as well as new methods of application.

The development of aerial spray application equipment has been more extensive than that of ground equipment. There is a very definite need, however, for the development of improved ground equipment for use against specific kinds of pests, particularly in areas where aerial applications are not practical.

Messenger $(1964,1965)$ reported on the successful use of low-volume (LV) aerial spray applications of technical, undiluted materials to suppress adult populations of the boll weevil, cereal leaf beetle, and beet leafhoppers.

Skoog et al. (1965) found that ultra-low-volume (ULV) aeria? spraying of dieldrin and malathion provided good control of rangeland grasshoppers.

Burgess (1965) achieved effective control of rangeland grasshoppers, cereal leaf beetle, and boll weevil using LV aerial spray applications.

Wilson et. al. (1965) reported effective control of cereal leaf beetle adults with aerial, LV concentrate spray applications.

Adkisson (1966) was successful in reducing diapausing boll weevil populations with LV sprays applied by aircraft.

Adair et al. (1967) reported that LV spray concentrates of commonly used insecticides, applied by aircraft, gave adequate control of cotton insects.

MacCuaig (1966) emphasized the effectiveness of ULV aerial sprays in desert locust control. The concentrate of malathion (LV) was more effective than dilute mixtures previously used. 
Niemczyk et al. (1967), using ULV aerial sprays, and Pass and Knapp (1966), applying LV aerial sprays, obtained fairly satisfactory control of the alfalfa weevil.

Other workers have been using ground equipment for the dispersal of LV and ULV spray mixtures. Bennett and Luttrell (1965) used LV applications of several insecticides to achieve excellent alfalfa weevil larval control for seven days.

Burt et al. (1966), Thomas (1966), and Cleveland et al. (1966) successfully controlled the cotton boll weevil in experiments using undiluted pesticides applied with ground equipment.

Harrell et al. (1966, 1967), using ground equipment to apply LV sprays in sweetcorn, obtained satisfactory ear-worm control.

Harrell and Leuck (1967) achieved equal or better control of soybean insects with ULV sprays applied with ground equipment than was obtained with dilute insecticides applied conventionally.

In the alfalfa weevil control experiments described in this bulletin, LV and ULV ground equipment was used to apply the sprays. The experimental areas were located in the Eastern Panhandle and in northern areas of the State. In general, weevil populations were high in these communities.

In 1965,15 acres were involved in these experiments; in 1966 there were 164 acres, and in 1967, 49 acres.

\section{Methods and Materials}

ULV applications were made in 1965 with an air-siphon sprayer constructed in the machine shop of the West Virginia University Reedsville Experiment Farm. The machine (hereafter referred to as Reedsville sprayer) consisted of an air compressor joined with an air storage tank which was connected by means of a flexible hose to a manifold equipped with a pressure regulator and gage and two Spraying Systems fluid nozzles No. 35100 and air nozzles 120432. The ULV insecticides were air-siphoned from containers mounted directly under the delivery nozzles. The spray system was enclosed in a canvas and plastic canopy to minimize effects of the wind on the fine droplets. The entire unit was mounted on a tractor-drawn trailer. The spray pattern of the nozzles converged and covered a swath about nine feet wide. Figure 1 shows some of the details of construction and of the sprayer in operation.

A Buffalo, turbine-type dilute sprayer was used to apply insecticides on some of the plots in 1965 (Figure 2). 


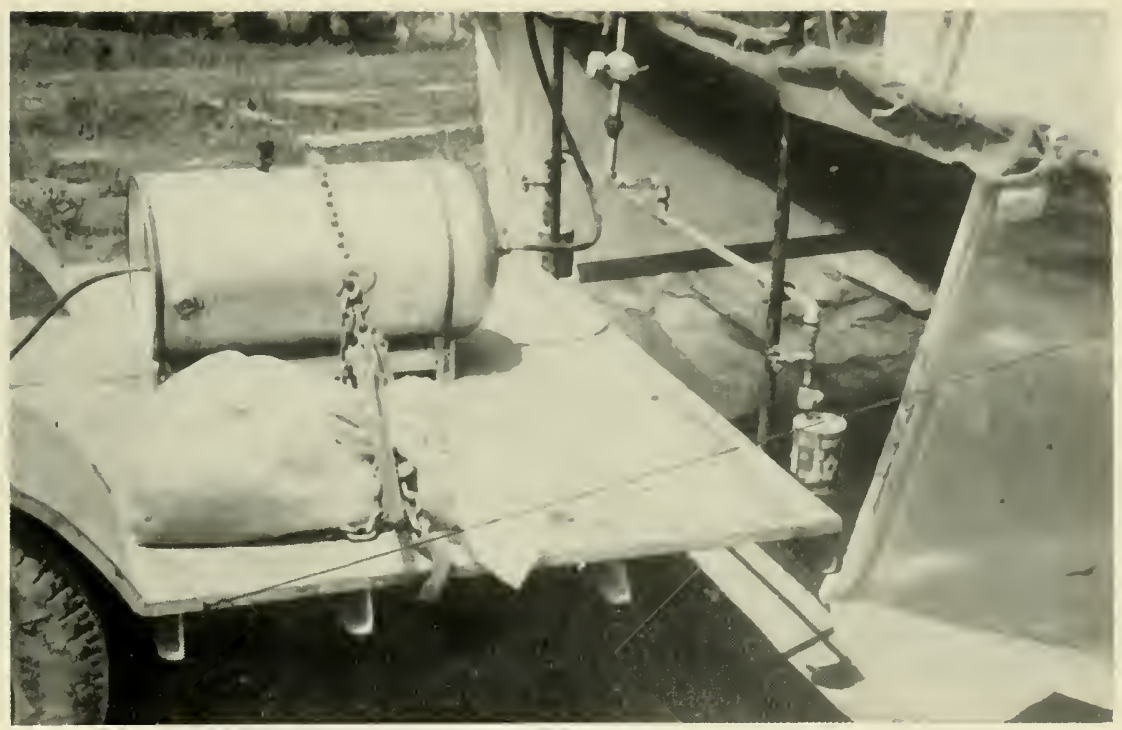

Figure 1. Air siphon type ULV sprayer used in the 1965 experiments.

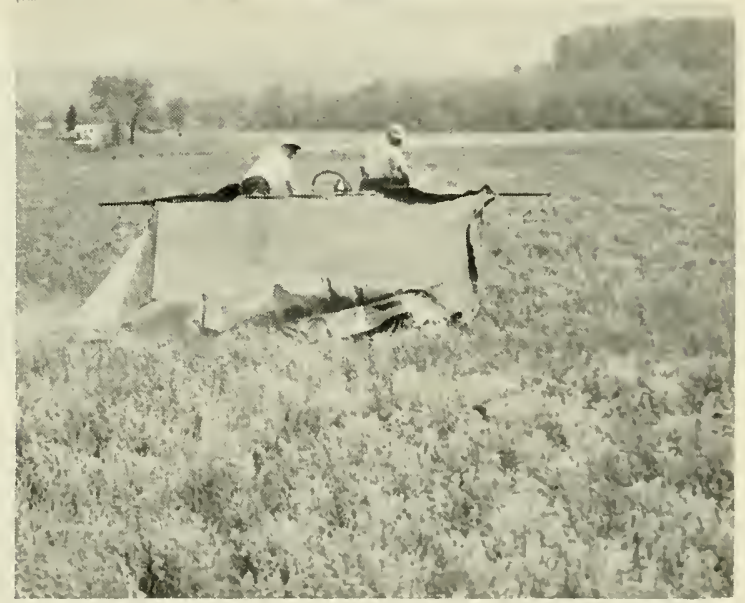

An Econo-Mist orchard sprayer, converted for use as a forage crop sprayer, was used to apply LV and ULV sprays on alfalfa weevil control plots in 1966. Figure 3 illustrates the type of machine and its operation. A 40-foot swath was uniformly covered with droplets 50-75 microns in size. The spray pattern was directed by hydraulic controls. When ULV sprays were applied, the feeder line was placed directly in the container of concentrate insecticide. The sprayer was equipped wtih two metering pumps (six cylinders). One could be adjusted to deliver insecticides within 


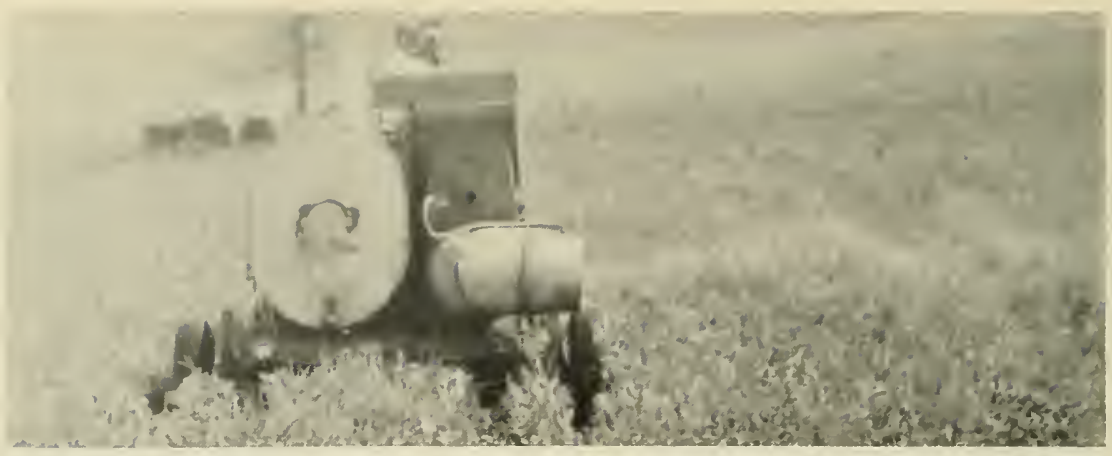

Figure 2. Buffalo turbine type dilute sprayer used in the $\mathbf{1 9 6 5}$ experiments.

the range of $8 \mathrm{oz}$ to $128 \mathrm{oz}$ of spray material per acre; the other could be adjusted to deliver one to four gallons of spray mixture per acre. The sprayer was pulled with a large tractor at speeds between 3.5 and $4 \mathrm{mph}$.

The Reedsville sprayer used in 1965 was remodeled for use in the 1966 experimental program. The canopy frame was redesigned with lateral extensions which would telescope to permit easy access through gates. The ends and front of the frame were enclosed with plywood and the remainder of the spray chamber was covered with heavy canvas. The number of nozzles was increased to four. The nozzles (Spraying Systems air nozzles No. 67147 and fluid nozzles No. 2050), each with a container of insecticide, were placed on the outside of the spray chamber. The spray was directed into the spray chamber through openings in the forward wall. The air compressor, which was driven by power take-off, was installed on the back of the tractor. The spray application covered a swath 20 feet wide and could be effectively pulled at tractor speeds of 3 to $5 \mathrm{mph}$. The insecticide application rates were obtained by varying the ground speed and air pressure on the system. Figure 4 depicts some of the details described.

The remodeled 1966 Reedsville sprayer was further modified for the 1967 ULV spraying program (Figure 5). The air-siphon nozzles and insecticide containers were removed and replaced with a central insecticide tank which was pressurized directly from the air storage tank, and four pressurized nozzles (same as 1966) were installed rather than air-siphon type nozzles. Pressurizing the insecticide tank was necessary in order to achieve equal distribution of insecticide to each of the four nozzles. When each 

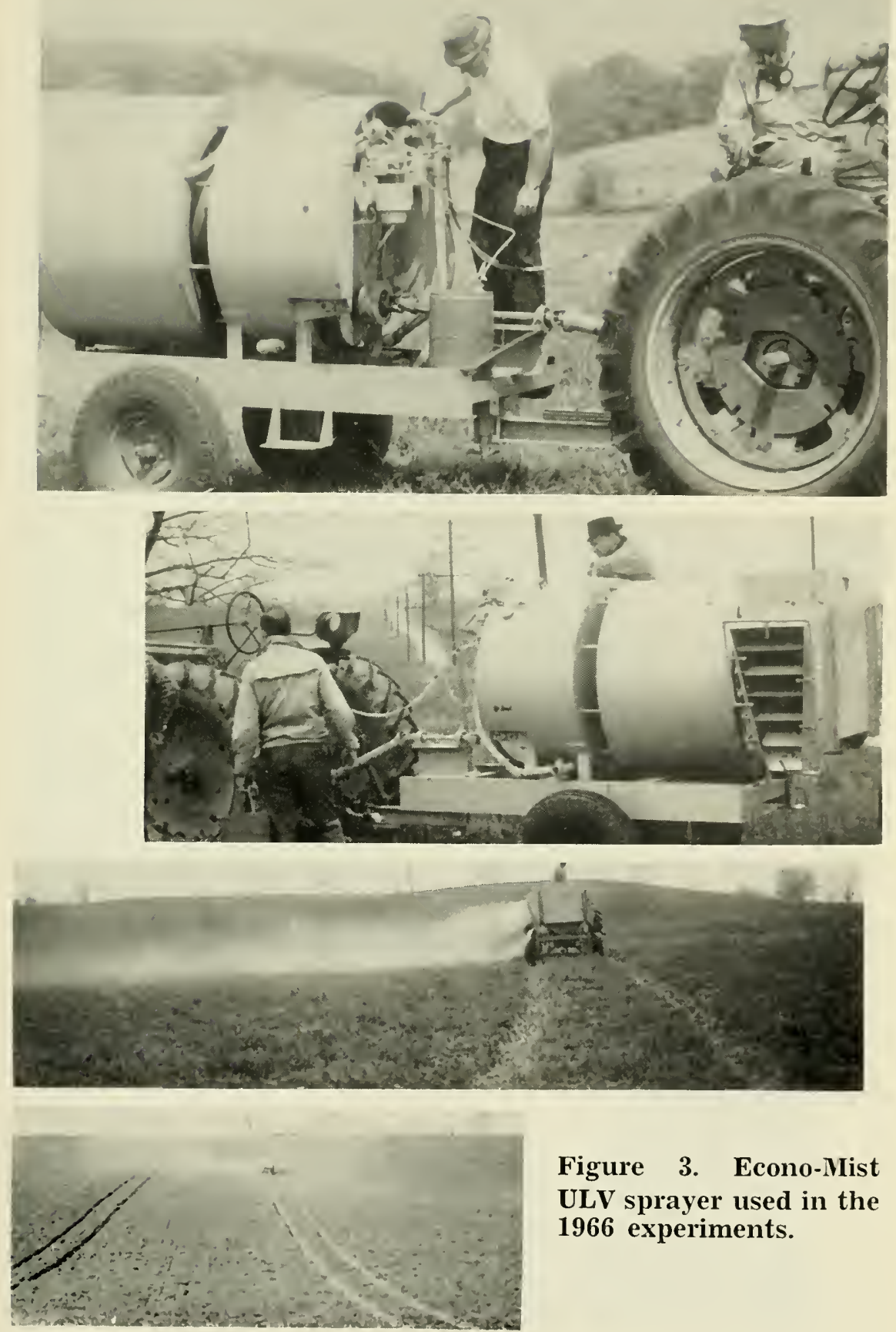

Figure 3. Econo-Mist ULV sprayer used in the 1966 experiments. 

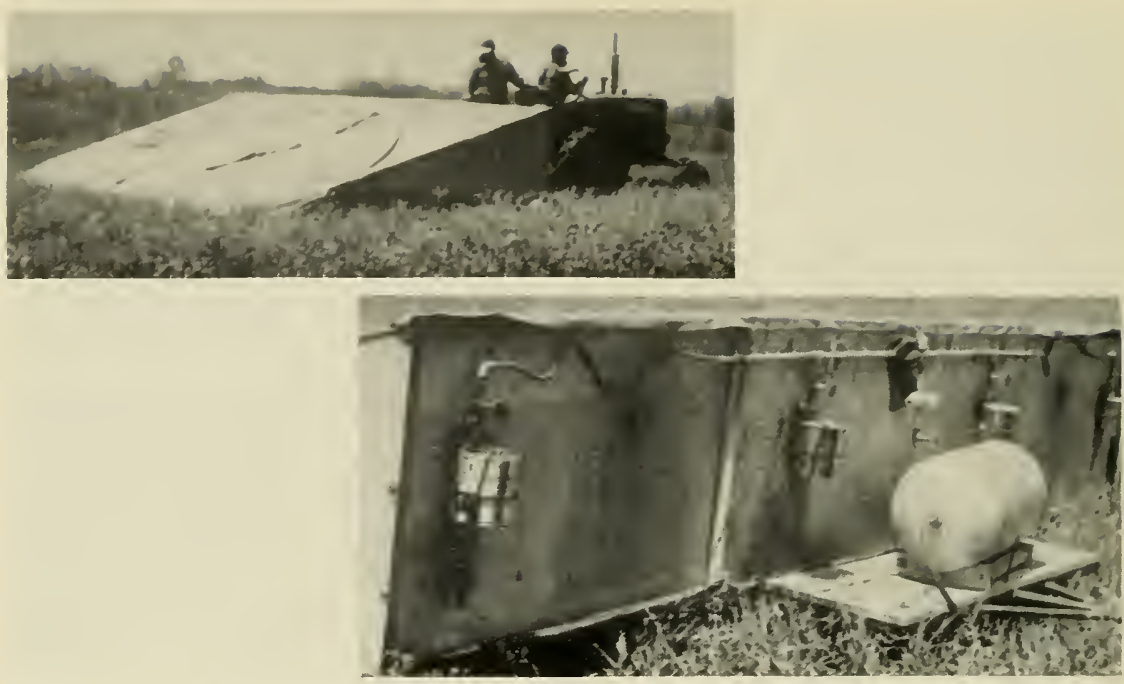

Figure 4. Modification of the 1965 model Reedsville ULV sprayer used in the 1966 weevil control experiments.

nozzle was equipped with a separate insecticide container (1965 and 1966) and all nozzles shared a common air-line, the pressures were not equal at all nozzle outlets; this affected the spray pattern and droplet dispersal. The change in design for the 1967 program largely corrected this problem.

Fifteen different chemical treatments were applied experimentally in this program (1965-67); 12 of these materials have common names and are listed in Tables 1 to 6 . The three proprietary materials used were SD-7438, S, S'-benzylidene bis-0-0dimethyl phosphorodithioate); American Cyanamid 47470, 2-(diethoxyphosphinylimino) - 4 - methyl - 1,3-dithiolane; and Imidan $\AA$, N - (mercaptomethyl phthalimide S - (O-O-dimethylphosphorodithioate).

\section{Results and Discussion}

In 1965 ULV and dilute sprays were applied on plots on the Reedsville farm in mature (for first cutting) alfalfa which had been badly damaged by larval feeding. Malathion sprays applied with the Reedsville ground sprayer gave perfect adult control seven days post-treatment. All of the spray applications (ULV and dilute) gave excellent larval control one and two weeks posttreatment (Table 1). 

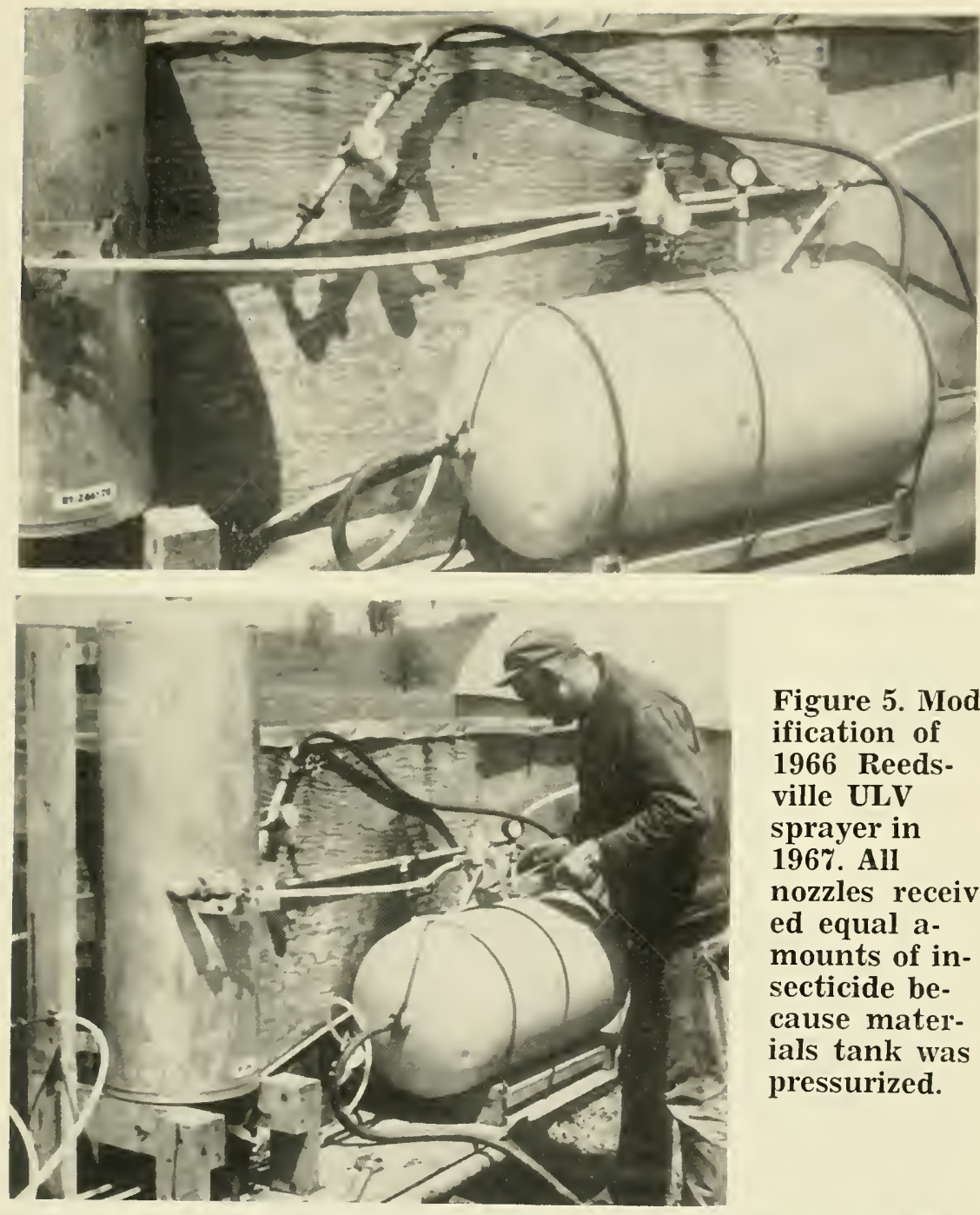

Figure 5. Modification of 1966 Reedsville ULV sprayer in 196\%. All nozzles received equal amounts of insecticide because materials tank was pressurized.

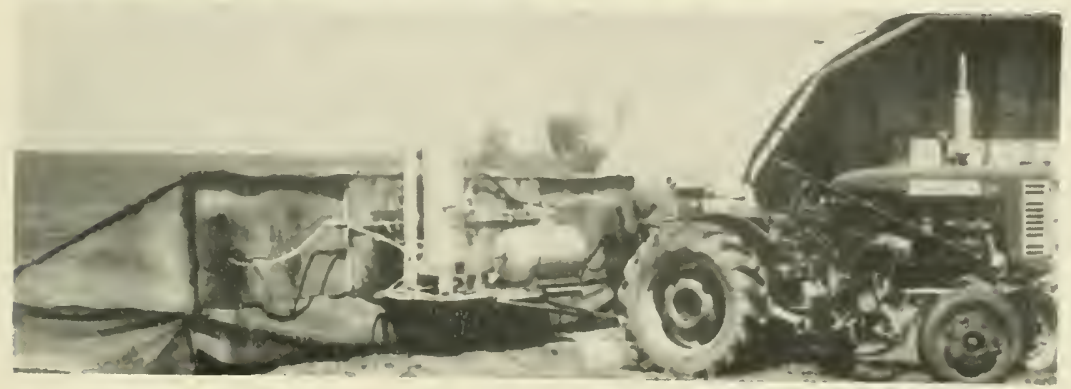


TABLE 1

Comparative effectiveness of foliar treatment applied with airblower (dilute) and air-siphon (ultra-low-volume) ground sprayers for the control of the alfalfa weevil (1965).

\begin{tabular}{|c|c|c|c|c|c|}
\hline \multicolumn{3}{|c|}{ Adult Weevils } & \multicolumn{3}{|c|}{ Weevil Larvae } \\
\hline $\begin{array}{l}\text { Treat- } \\
\text { ments }^{a}\end{array}$ & $\begin{array}{l}\text { Comp. } \\
\text { Effec- } \\
\text { tiveness }^{b}\end{array}$ & $\% \mathbf{K i l l} * *$ & $\begin{array}{l}\text { Treat- } \\
\text { ments }^{a}\end{array}$ & $\begin{array}{c}\text { Comp. } \\
\text { Effec- } \\
\text { tiveness }^{b}\end{array}$ & $\%$ Kill** \\
\hline \multicolumn{6}{|c|}{$\begin{array}{l}\text { REEDSVILLE FARM (Plots 1.25A x 2) } \\
\text { (Coll. 5-21-65) }\end{array}$} \\
\hline 1 & a & 100.0 & 6 & a & 98.0 \\
\hline 2 & $\mathrm{a}$ & 100.0 & 2 & $a$ & 98.0 \\
\hline 3 & a & 100.0 & 5 & a & 95.0 \\
\hline 4 & b & 0.0 & 3 & $a b$ & 95.0 \\
\hline 5 & b & $0.0^{\mathrm{e}}$ & 1 & $\mathrm{~b}$ & 91.0 \\
\hline 6 & $\mathrm{~b}$ & $0.0^{\mathrm{c}}$ & 4 & $\mathrm{c}$ & 0.0 \\
\hline \multicolumn{6}{|c|}{ (Coll. 5-28-65) } \\
\hline 5 & a & 48.0 & 6 & a & 99.0 \\
\hline 4 & $\mathrm{~b}$ & 0.0 & 2 & $\mathrm{a}$ & 98.0 \\
\hline 6 & $\tilde{b}$ & $0.0^{\mathrm{c}}$ & $\overline{5}$ & $a b$ & 98.0 \\
\hline 3 & $\mathrm{~b}$ & $0.0^{\circ}$ & 3 & $a b$ & 97.0 \\
\hline 1 & b & $0.0^{\mathrm{c}}$ & 1 & $a b$ & 97.0 \\
\hline 2 & $\mathrm{~b}$ & $0.0^{\mathrm{c}}$ & 4 & $c$ & 0.0 \\
\hline
\end{tabular}

ac Treatments: (AS = air-siphon, ULV sprayer, $30 \mathrm{psi}, 3 \mathrm{mph}$; used in treating all of Reedsville Farm plots in 1966 except as otherwise indicated; $\mathrm{AB}=$ Buffalo air-blower sprayer; treatments applied 5-20-65)

1. Malathion, LV $(3.0 \mathrm{lb} / \mathrm{A})$, AS $(39 \mathrm{oz} / \mathrm{A})$

2. Malathion, LV (2.0) AS $(26 \mathrm{oz} / \mathrm{A})$

3. Malathion, EC (2.0) AS $(52 \mathrm{oz} / \mathrm{A})$

4. Untreated (geometric average adult wevil comt 8.0; larval count 1739.0)

5. Malathion, EC (1.75) AB (20 gal/A)

6. Azinphosmethyl, EC (0.4) AB (20 gal/A)

"Duncan's Multiple Range Test at level indicated for $\log (\mathrm{N}+\mathrm{I})$ of the data; antilog of data means -1 is presented as the gcometric average count for 25 swceps. Treatments sharing a letter in common do not differ in effectiveness.

'The aberrant data concerning adult weevil counts is mainly because of the scarcity of weevils at this time of the season. Negative control (more specimens in treated than in untreated plots) is acknowledged by $0.0 \%$ to inclicate lack of control.

**5.0 per cent level of significance.

ULV sprays applied as stubble treatment on the Reedsville farm all gave economic control (80 per cent or more reduction) of adult weevils one week post-treatment. Sun Oil 7EL and TSEL gave economic control two and three weeks post-treatment and malathion two weeks post-treatment. All treatments produced excellent larval control results one and two weeks post-treatment, 
Comparative effectiveness of concentrate (ULV) sprays applied with ground equipment as stubble treatment to control the alfalfa weevil (1965).

\begin{tabular}{|c|c|c|c|c|c|}
\hline \multicolumn{3}{|c|}{ Adult Weevils } & \multicolumn{3}{|c|}{ Weevil Larvae } \\
\hline $\begin{array}{l}\text { Treat- } \\
\text { ments }^{a}\end{array}$ & $\begin{array}{l}\text { Comp. } \\
\text { Effec- } \\
\text { tiveness }^{b}\end{array}$ & $\%$ Kill*** & $\begin{array}{l}\text { Treat- } \\
\text { ments }^{a}\end{array}$ & $\begin{array}{c}\text { Comp. } \\
\text { Effec- }^{\text {tiveness }}\end{array}$ & $\% \mathbf{K i l l}^{* *}$ \\
\hline \multicolumn{6}{|c|}{$\begin{array}{l}\text { REEDSVILLE FARM (Plots } 2 \mathrm{~A} \times 2) \\
\text { (Coll. } 7-1-65)\end{array}$} \\
\hline 5 & a & 100.0 & 5 & a & 100.0 \\
\hline 4 & $\mathrm{a}$ & 100.0 & 4 & a & 100.0 \\
\hline 3 & $a$ & 100.0 & 3 & $\mathrm{a}$ & $100 \cdot 0$ \\
\hline 2 & $a$ & 100.0 & 2 & $a$ & 100.0 \\
\hline 1 & $\mathrm{~b}$ & 83.0 & 1 & $\mathrm{~b}$ & 85.0 \\
\hline 6 & $\mathrm{c}$ & 0.0 & 6 & $\mathrm{c}$ & 0.0 \\
\hline \multicolumn{6}{|c|}{ (Coll. $7-7-65)$} \\
\hline 3 & a & 100.0 & 5 & a & 100.0 \\
\hline 4 & $\mathrm{~b}$ & 89.0 & 3 & $\mathrm{a}$ & 100.0 \\
\hline 1 & $\mathrm{~b}$ & 89.0 & 2 & $\mathrm{a}$ & 100.0 \\
\hline 5 & $\mathrm{bc}$ & 78.0 & 1 & $\mathrm{a}$ & 100.0 \\
\hline 2 & $\mathrm{c}$ & 67.0 & 4 & a & 90.0 \\
\hline 6 & d & 0.0 & 6 & $\mathrm{~b}$ & 0.0 \\
\hline \multicolumn{6}{|c|}{ (Coll. y-15-65) } \\
\hline 4 & $\mathrm{a}$ & 100.0 & 5 & a & 96.0 \\
\hline 3 & $\mathrm{a}$ & 100.0 & 1 & a & 96.0 \\
\hline 5 & $a b$ & 75.0 & 2 & $a b$ & 92.0 \\
\hline 2 & $a b$ & 75.0 & 4 & $a b$ & 86.0 \\
\hline 1 & $\mathrm{~b}$ & 25.0 & 3 & $a b$ & 54.0 \\
\hline 6 & $\mathrm{~b}$ & 0.0 & 6 & $\mathrm{~b}$ & 0.0 \\
\hline
\end{tabular}

"Treatments: (applied 3 days after hay removal; 6-24-65)

1. Malathion, $L V(0.5)(6-7 \mathrm{oz} / \mathrm{A})$

2. Azinphosmethyl, ULV (0.75) (48 o7/A)

3. 7EL (Sun Oil) (1 G/A) (128 oz/A)

4. TSEL, (Sun Oil) (1 G/A) (128 oz/A)

5. Malathion, EC (1.0) $(26 \mathrm{oz} / \mathrm{I})$

6. Untreated (geonetric average adult wecvil count 8.0 ; larval connt 21.0)

'Duncan's Multiple Range Test at level indicated for $\log (\mathrm{N}+1)$ of the data: $\operatorname{antilog}$ of data means -1 is presented as the geometric average count for 25 swecps. Treatments sharing a letter in common do not differ in effectireness.

**5.0 per cent level of significance

with the exception of 7EL which was effective for three weeks (Table 2).

Plots on the Widmyer farm, in the 1966 program, were treated with the modified Econo-Mist sprayer. The fall treatments were 
TABLE 3

Comparative effectiveness of alfalfa weevil control with concentrate (ULV) spray foliar treatments applied with ground equipment (1966).

\begin{tabular}{|c|c|c|c|c|c|}
\hline \multicolumn{3}{|c|}{ Adult Weevils } & \multicolumn{3}{|c|}{ Weevil Larvae } \\
\hline $\begin{array}{l}\text { Treat- } \\
\text { ments }^{a}\end{array}$ & $\begin{array}{c}\text { Comp. } \\
\text { Effec- } \\
\text { tiveness }^{b}\end{array}$ & $\%$ Kill $* * *$ & $\begin{array}{l}\text { Treat- } \\
\text { ments }^{a}\end{array}$ & $\begin{array}{c}\text { Comp. } \\
\text { Effec- } \\
\text { tiveness }^{b}\end{array}$ & $\% \mathbf{K i l l} * *$ \\
\hline \multicolumn{6}{|c|}{$\begin{array}{l}\text { A-WIDMYER FARM (Plots 2.25A x 2) } \\
\text { (Coll. 5-1-66) }\end{array}$} \\
\hline 4 & a & 100.0 & 7 & a & 88.0 \\
\hline 6 & $a b$ & 81.0 & 2 & b & 74.0 \\
\hline 2 & $a b$ & 66.0 & 6 & b & 67.0 \\
\hline 5 & $a b$ & 54.0 & 5 & $\mathrm{bc}$ & 50.0 \\
\hline 3 & $a b$ & 54.0 & 1 & $\mathrm{bc}$ & 49.0 \\
\hline 1 & $a b$ & 54.0 & 3 & $\mathrm{bc}$ & 34.0 \\
\hline 8 & $\mathrm{~b}$ & 0.0 & 4 & $\mathrm{c}$ & 9.0 \\
\hline 7 & $\mathrm{~b}$ & $0.0^{\mathrm{c}}$ & 8 & c & 0.0 \\
\hline
\end{tabular}

Table 3-A Treatments: (Econ-o-Mist ULV sprayer; applied as indicated)

1. $7 \mathrm{~N}$ (Sun Oil) $(4 \mathrm{G} / \mathrm{A})+$ Genite, $\mathrm{EC}(1.0), 3-22-66$ (4.5 $\mathrm{gal} / \mathrm{A})$

2. $7 \mathrm{~N}(4 \mathrm{G}) 3-22-66(4 \mathrm{gal} / \mathrm{A})$

3. 9lEL (Sun Oil) (4G) + Malathion, LV (0.3), 3-22-66 (4 gal/A)

4. 9IEL (Naphthenic) (4G), 3-22-66 (4 gal/A)

5. 9IEL $(4 \mathrm{G})+$ DMSO $(1.0 \% / \mathrm{A}), 10-12-65(4 \mathrm{gal} / \mathrm{A})$

6. $91 \mathrm{~N}$ (Sun Oil) (4G), 11-16-65 (4 gal/A)

7. 7EL (Sun Oil) (4G) + Genite, EC (1.0), 3-22-66 (4.5 gal/A)

8. Untreated (geometric average adult weevil count 9.0; larvel count 1199.0)

\section{B-WIDMYER FARM (Plots $1.6 \mathrm{~A} \times 2$ )}

(Coll, 5-15-66)

$\begin{array}{rrrrlr}10 & \mathrm{a} & 100.0 & 1 & \mathrm{a} & 96.0 \\ 6 & \mathrm{a} & 100.0 & 6 & \mathrm{a} & 94.0 \\ 5 & \mathrm{a} & 100.0 & 2 & \mathrm{ab} & 93.0 \\ 1 & \mathrm{a} & 100.0 & 7 & \mathrm{ab} & 92.0 \\ 9 & \mathrm{a} & 100.0 & 5 & \mathrm{ab} & 91.0 \\ 7 & \mathrm{a} & 92.0 & 9 & \mathrm{ab} & 90.0 \\ 3 & \mathrm{a} & 92.0 & 8 & \mathrm{ab} & 90.0 \\ 2 & \mathrm{a} & 92.0 & 10 & \mathrm{abc} & 89.0 \\ 8 & \mathrm{a} & 81.0 & 3 & \mathrm{bc} & 88.0 \\ 4 & \mathrm{a} & 81.0 & 4 & \mathrm{c} & 82.0 \\ 11 & \mathrm{~b} & 0.0 & 11 & \mathrm{~d} & 0.0\end{array}$

${ }^{2}$ Table 3-B Treatments: (Econ-o-Mist ULV sprayer; applied 4-19-66)

1. Azinphosmethyl, EC $(0.8)(50 \mathrm{oz} / \mathrm{A})$

2. $\mathrm{SD}-7438, \mathrm{EC}(0.8)(50 \mathrm{oz} / \mathrm{A})$

3. Malathion, EC $(0.75)(20 \mathrm{oz} / \mathrm{A})$

4. Malathion, LV $(0.4)(6-7 \mathrm{oz} / \mathrm{A})$

5. Azinphosmethyl, ULV (0.75)

$(48 \mathrm{oz} / \mathrm{A})$

6. SD-7438, EC (1.0) + 91EL (Sun Oil) (4 G/A) $(4.5 \mathrm{gal} / \mathrm{A})$
7. Azinphosmethyl, EC $(0.8)(50 \mathrm{oz} / \mathrm{A})$

8. Malathion. $\mathrm{LV}(0.4)(6-7 \mathrm{oz} / \mathrm{A})$

9. Malathion, EC $(0.75)(2007 / A)$

10. Azinphosmethrl, $\mathrm{ULV}^{\top}(0.75)$ $(48 \mathrm{o} / \mathrm{A})$

11. Untreated (geometric average adnlt weevil count 5.0; larval count 1199.0) 
C-FRENCH FARM (Plots 2A $\mathrm{x}$ 2)

(Coll. 5-10-66)

$\begin{array}{rlrllr}2 & \mathrm{a} & 100.0 & 2 & \mathrm{a} & 100.0 \\ 3 & \mathrm{ab} & 70.0 & 3 & \mathrm{ab} & 55.0 \\ 1 & \mathrm{ab} & 70.0 & 4 & \mathrm{ab} & 44.0 \\ 4 & \mathrm{~b} & 0.0 & 1 & \mathrm{~b} & 40.0 \\ 5 & \mathrm{~b} & 0.0 & 5 & \mathrm{c} & 0.0\end{array}$

(Coll. 5-19-66)

$\begin{array}{rlrrrr}2 & \mathrm{a} & 84.0 & 3 & \mathrm{a} & 48.0 \\ 3 & \mathrm{ab} & 56.0 & 2 & \mathrm{a} & 47.0 \\ 4 & \mathrm{ab} & 50.0 & 1 & \mathrm{a} & 32.0 \\ 1 & \mathrm{~b} & 33.0 & 4 & \mathrm{~b} & 20.0 \\ 5 & \mathrm{~b} & 0.0 & 5 & \mathrm{~b} & 0.0\end{array}$

"Table 3-C Treatments: (Econ-o-Mist ULV sprayer; applied 5-5-66)

1. Malathion, EC $(1.25)+$ Methoxychlor, EC (1.25) (113 oz/A)

2. Azinphosmethyl, ULV (0.75) (48 oz/A)

3. Malathion, EC (1.0) + Methoxychlor, EC (1.0) $90 \mathrm{or} / \mathrm{A})$

4. Malathion, LV $(0.8)(10 \mathrm{oz} / \mathrm{A})$

5. Untreated (geometric average adult weevil count 6.0 ; larval count 334.0)

'Duncan's Multiple Range Test at level indicated for $\log (\mathrm{N}+1)$ of the data; antilog of data means -1 is presented as the geometric average count for 25 sweeps. Treatments sharing a letter in common do not differ in effectiveness.

'The aberrant data concerning adult weevil counts is mainly because of the scarcity of weerils at this time of the season. Negative control (more specimens in treated than in untreated plots) is acknowledged by $0.0 \%$ to indicate lack of control.

** 5.0 per cent level of significance.

***10.0 per cent level of significance.

directed mainly at ovipositing adults and eggs and the spring treatments were meant for early larval stages and early emerging adult weevils. (Ninety-five per cent of the fall-laid eggs normally hatch in West Virginia by mid-March.) The population sampling date $(5 / 1 / 66)$ was six and seven months after fall treatments and five weeks after spring treatments depending upon the treatment and date of application (Table 3-A). Only the Sun Oil 91EL treatment (3-22-66 application) and the 91N treatment (applied 11-16-65) gave satisfactory adult reduction and only 7EL plus Genite (applied 3-22-66) produced economic control of the larvae.

In other plots on the Widmyer farm treated with ULV applications (Econo-Mist sprayer) all treatments gave economic control (80 per cent or more population reduction) (Table $3-\mathrm{B}$ ).

ULV applications on the French farm were also applied with the Econo-Mist sprayer in 1967. Azinphosmethyl ULV was the most effective treatment against the adult one and two weeks post-treatment and against the larvae one week post-treatment. No treatment gave economic larval reduction nine days post-treat- 
Comparative effectiveness of alfalfa weevil control with concentrate (ULV) air-siphon foliar spray applications (1966).

\begin{tabular}{|c|c|c|c|c|c|}
\hline \multicolumn{3}{|c|}{ Adult Weevils } & \multicolumn{3}{|c|}{ Weevil Larvae } \\
\hline $\begin{array}{l}\text { Treat- } \\
\text { ments }\end{array}$ & $\begin{array}{l}\text { Comp. } \\
\text { Effec- } \\
\text { tiveness }^{b}\end{array}$ & $\%$ Kill & $\begin{array}{l}\text { Treat- } \\
\text { ments }\end{array}$ & $\begin{array}{c}\text { Comp. } \\
\text { Effec- }^{\text {tiveness }}\end{array}$ & $\%$ Kill \\
\hline \multicolumn{6}{|c|}{$\begin{array}{c}\text { A-REEDSVILLE FARMI } \\
\text { (Coll. } 6-17-66)\end{array}$} \\
\hline 1 & a & 92.0 & 1 & a & 52.0 \\
\hline 2 & $\mathrm{~b}$ & $0.0 \% *$ & 2 & b & $0.0 *$ \\
\hline \multicolumn{6}{|c|}{ (Coll. 6-24-66) } \\
\hline 1 & a & 60.0 & 1 & a & 60.0 \\
\hline & a & 0.0 & 2 & a & 0.0 \\
\hline
\end{tabular}

${ }^{a}$ Table 4-A Treatments: (applied 6-10-66)

1. SD-7438, EC (0.5) + Methyl parathion, EC (0.5) (32 oz/A)

2. Untreated (geometric average adult weeril count 45.0; larval count 226.0)

\begin{tabular}{|c|c|c|c|c|c|}
\hline & & $\begin{array}{l}\text { FARMI } \\
\text { (Coll. }\end{array}$ & 5) & ts & \\
\hline 1 & a & 100.0 & 1 & a & 41.0 \\
\hline 2 & $b$ & $0.0 \% \div$ & 2 & a & $0.0 \%$ \\
\hline
\end{tabular}

(Coll. 6-8-66)

$\begin{array}{cccccc}1 & \mathrm{a} & 62.0 & 1 & \mathrm{a} & 77.0 \\ 2 & \mathrm{~b} & 0.0 * * * & 2 & \mathrm{~b} & 0.0 *\end{array}$

"Table 4-B Treatments: (applied 5-24-66)

1. SD-7438, EC $(0.25)$ + Methrl parathion, EC $(0.25)(32 \mathrm{oz} / \mathrm{A})$

2. Untreated (geometric average adult weevil count 12.0; larval count 500.0)

C-REEDSVILLE FARM (A1, S2, and 3) (Plots 3.5A x 2) (Coll. 6-1-66)

$\begin{array}{cccccc}1 & \mathrm{a} & 100.0 & 1 & \mathrm{a} & 92.0 \\ 2 & \mathrm{~b} & 0.0 * * & 2 & \mathrm{~b} & 0.0 * *\end{array}$

$\begin{array}{rrrrrr}1 & \mathrm{a} & 12.0 & 1 & \mathrm{a} & 92.0 \\ 2 & \mathrm{a} & 0.0 & 2 & \mathrm{~b} & 0.0^{* *}\end{array}$

aTable 4-C Treatments: (applied 5-25-66)

1. Malathion, LV (0.4) (6-7 oz/A)

2. Untreated (geometric average adult weevil count 12.0; larral count 500.0)

ment. In this field one application gave good protection against larval damage until the first cutting even though the per cent kill on the sampling dates is rather low (Table 3-C).

Plots on the Reedsville farm treated with the Reedsville 
D-REEDSVILLE FARM (A4, S2) (Plots 1.2A x 2)

(Coll. 5-31-66)

$\begin{array}{cccccc}1 & \mathrm{a} & 84.0 & 1 & \mathrm{a} & 80.0 \\ 2 & \mathrm{~b} & 0.0 * * * & 2 & \mathrm{~b} & 0.0^{* * *}\end{array}$

(Coll. 6-6-66)

$\begin{array}{cccccc}1 & \mathrm{a} & 84.0 & 1 & \mathrm{a} & 97.0 \\ 2 & \mathrm{~b} & 0.0 * * * & 2 & \mathrm{~b} & 0.0 * *\end{array}$

"Table 4D Treatments: (applicd 5-24-66)

1. SD-7438, EC $(0.75)(48 \mathrm{oz} / \mathrm{A})$

2. Untreated (geometric aserage adule wecvil count 12.0; larval count 500.0)

E-REEDSVILLE FARM (A1, S2 and 3) (Plots 3.3A x 2) (Coll. 6-17-67)

$\begin{array}{rrrrrr}1 & \mathrm{a} & 23.0 & 1 & \mathrm{a} & 99.0 \\ 2 & \mathrm{a} & 0.0 & 2 & \mathrm{~b} & 0.0 *\end{array}$

(Coll. 6-24-66)

$\begin{array}{rrrrrr}1 & \mathrm{a} & 80.0 & 2 & \mathrm{a} & 0.0 \\ 2 & \mathrm{a} & 0.0 & 1 & \mathrm{a} & 0.0^{\circ}\end{array}$

"Table 4-E Treatments: (applied 6-10-66)

1. Malathion, LV $(0.75)(10 \mathrm{oz} / \mathrm{A})$

2. Untreated (geometric average adult weevil count 45.0; larval count 357.0)

F-REEDSVILLE FARM (A4, S5) (Plots 1.2A x 2)

(Coll. 5-31-66)

$\begin{array}{cccccc}1 & \mathrm{a} & 80.0 & 2 & \mathrm{a} & 0.0 \\ 2 & \mathrm{~b} & 0.0 * * * & 1 & \mathrm{a} & 0.0^{\mathrm{c}}\end{array}$

(Coll. 6-6-66)

$\begin{array}{cccccc}1 & \mathrm{a} & 67.0 & 1 & \mathrm{a} & 33.0 \\ 2 & \mathrm{~b} & 0.0^{* *} & 2 & \mathrm{a} & 0.0 * *\end{array}$

aTable 4-F Treatments: (applied 5-24-66)

1. Malathion, LV $(0.75)(10 \mathrm{oz} / \mathrm{A})$

2. Untreated (geometric average adult weevil count 11.0; larval count 300.0)

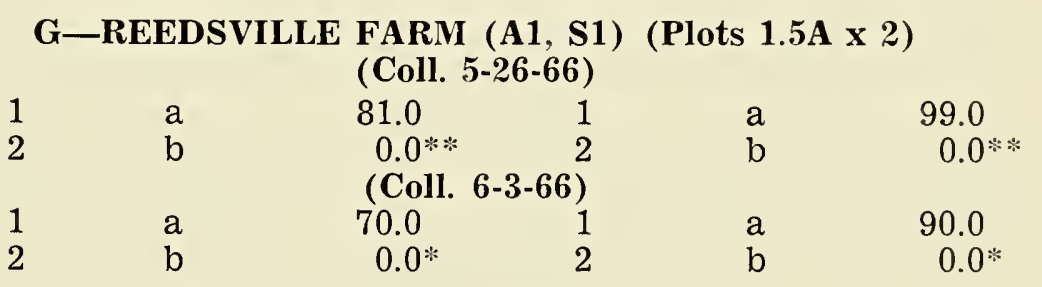

(Coll. 6-10-66)

$\begin{array}{cccccc}1 & \mathrm{a} & 72.0 & 1 & \mathrm{a} & 82.0 \\ 2 & \mathrm{~b} & 0.0 * & 2 & \mathrm{~b} & 0.0^{*}\end{array}$

"Table 4-G Treatments: (applied 5-20-66)

1. Ainphosmcthyl, EC (0.75) (50 oz/A)

2. Untreated (geometric arerage adult weevil comt 4.0: larval count 648.0)

(Continued) 
TABLE 4 (Continued)

\begin{tabular}{|c|c|c|c|c|c|}
\hline \multicolumn{3}{|c|}{ Adult Weevils } & \multicolumn{3}{|c|}{ Weevil Larvae } \\
\hline $\begin{array}{l}\text { Treat- } \\
\text { ments }\end{array}$ & $\begin{array}{l}\text { Comp. } \\
\text { Effec- } \\
\text { tiveness }^{b}\end{array}$ & $\%$ Kill & $\begin{array}{l}\text { Treat- } \\
\text { ments }\end{array}$ & $\begin{array}{c}\text { Comp. } \\
\text { Effec- }^{-} \\
\text {tiveness }^{b}\end{array}$ & $\%$ Kill \\
\hline \multicolumn{6}{|c|}{$\begin{array}{c}\text { H-REEDSVILLE FARM (A4.S4) (Plots 1.2A x 2) } \\
\text { (Coll. 5-31-66) }\end{array}$} \\
\hline $\begin{array}{l}1 \\
2\end{array}$ & $\begin{array}{l}\mathrm{a} \\
\mathrm{a}\end{array}$ & $\begin{array}{l}50.0 \\
0.0 * *\end{array}$ & $\begin{array}{l}1 \\
2\end{array}$ & $\begin{array}{l}\mathrm{a} \\
\mathrm{b}\end{array}$ & $\begin{array}{l}46.0 \\
0.0 \% * \%\end{array}$ \\
\hline \multicolumn{6}{|c|}{ (Coll. 6-6-66) } \\
\hline $\begin{array}{l}2 \\
1\end{array}$ & $\begin{array}{l}a \\
a\end{array}$ & $\begin{array}{l}0.0 \\
0.0^{\circ}\end{array}$ & $\begin{array}{l}1 \\
2\end{array}$ & $\begin{array}{l}a \\
b\end{array}$ & $\begin{array}{l}89.0 \\
0.0 * *\end{array}$ \\
\hline
\end{tabular}

${ }^{2}$ Table 4-H Treatments: (applied 5-24-66)

1. Malathion, EC (1.0) $(26 \mathrm{oz} / \mathrm{A})$

2. Untreated (geometric average aduit weevil count 23.0; larval count 500.0)

\section{I-REEDSVILLE FARM (A2 S2, 3, and 5) (Plots 1A x 2)} (Coll. 6-1-66)

$\begin{array}{cccccc}1 & \mathrm{a} & 67.0 & 1 & \mathrm{a} & 89.0 \\ 2 & \mathrm{a} & 0.0 * * & 2 & \mathrm{~b} & 0.0^{* *}\end{array}$

(Coll. 6-8-66)

\begin{tabular}{cccccc}
1 & $\mathrm{a}$ & 77.0 & 1 & $\mathrm{a}$ & 94.0 \\
2 & $\mathrm{~b}$ & $0.0 * * *$ & 2 & $\mathrm{~b}$ & $0.0 * * *$ \\
\hline
\end{tabular}

${ }^{a}$ Table 4-I Treatments: (applied 5-25-66)

1. Malathion, LV (0.4) (6-7 oz/A)

2. Untreated (geometric average adult weevil count 12.0; larval count 400.0)

\begin{tabular}{cccccr} 
J-REEDSVILLE FARM & \multicolumn{1}{c}{ (A1, S5E) } & (Plots & 0.75A x 2) \\
(Coll. & 6-1-66) & & \\
1 & a & 67.0 & 1 & a & 68.0 \\
2 & b & $0.0 * *$ & 2 & a & 0.0
\end{tabular}

(Coll. 6-8-66)

\begin{tabular}{rrrrrr}
1 & $\mathrm{a}$ & 84.0 & 1 & $\mathrm{a}$ & 83.0 \\
2 & $\mathrm{~b}$ & $0.0 * * *$ & 2 & $\mathrm{a}$ & 0.0 \\
\hline
\end{tabular}

${ }^{2}$ Table 4-J Treatments: (applied 5-25-66)

1. Malathion, LV (0.4) (6-7 oz/A)

2. Untreated (geometric average adult weevil count 12.0; larval count 500.) 
K-REEDSVILLE FARM (A4, S3) (Plots 1.5A x 2)

(Coll. 5-31-66)

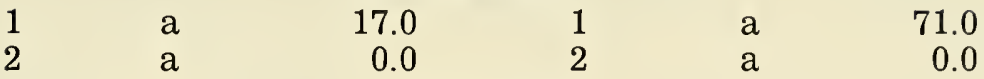

(Coll. 6-6-66)

$\begin{array}{cccccc}1 & \mathrm{a} & 45.0 & 1 & \mathrm{a} & 99.0 \\ 2 & \mathrm{~b} & 0.0^{* * *} & 2 & \mathrm{~b} & 0.0^{*}\end{array}$

"Table 4-K Treatments: (applied 5-24-66)

1. Azinphosmethyl, ULV $(0.75)(48 \mathrm{oz} / \Lambda)$

2. Untreated (geometric average adult count 12.0; larval count 518.0)

'Duncan's Multiple Range Test at level indicated for $\log (\mathbf{N}+1)$ of the clata; antilog of data means -1 is presented as the geometric average count for 25 sweeps. Treatments sharing a letter in common do not differ in effectiveness.

"The abcrrant data concerning adult weevil counts is mainly because of the scarcity of weevils at this time of the season. Negative control (more specimens in treated than in untreated plots) is acknowledged by $0.0 \%$ to indicatc lack of control.

*1.0 per cent level of significance.

** 5.0 per cent level of significance.

***10.0 per cent level of significance.

air-siphon ULV sprayer, using SD-7438 plus methyl parathion, produced significant, but not particularly good, weevil reductions (Table 4-A and B). Adjacent plots treated with malathion LV gave excellent adult reduction one week post-treatment and for one and two weeks post-treatment against larvae (Table 4-C). Malathion applied as ULV sprays in other plots on the Reedsville farm (Al, S2 and 3), (A4, S5), (A4, S4), (A1, S5E) produced rather erratic results in controlling adult and larval weevils (Table 4-E, F, H, and I).

In other plots on the Reedsville farm where ULV, SD-7438 spray was applied, economic reductions of adults and larvae were evident one and two weeks post-treatment (Table 4-D).

Azinphosmethyl ULV sprays (Plots $\mathrm{Al}, \mathrm{S} 1$ and $\mathrm{A} 4, \mathrm{~S} 3$ ) also produced erratic control results, but the effectiveness against larval stages was slightly better than with malathion sprays (Table 4-G and $\mathrm{K}$ ).

Alfalfa hay yield data taken from plots treated with ULV spray applications show that plots treated with concentrate sprays on the Widmyer and French farms produced more alfalfa than untreated fields (Table 5-A and B). The best aflalfa yield on the French farm resulted from the use of a mixture of malathion and methoxychlor. The best hay yield on the Widmyer farm was from azinphosmethyl plots.

In 1967, only the air-siphon-type ground sprayer was used to apply ULV treatments on the Reedsville Farm, and economic 
TABLE 5

Hay yield data from fields treated with ULV (undiluted) sprays (1966).

\section{Treatments \\ Total \\ Yield \\ A-FRENCH FARM-Field 1}

Alfalfa

Yield

Concentrate (undiluted) spray

Malathion $(23 \%)+$ Methoxychlor $(23 \%)$ (3 qts. $/ \mathrm{A})$

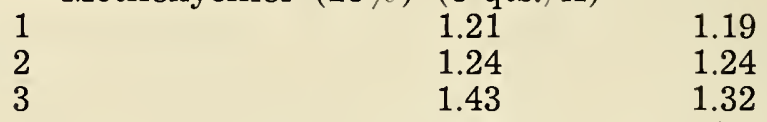

(av. 1.29) (av. 1.25)

Malathion, LV (57\%) (20 oz./A)

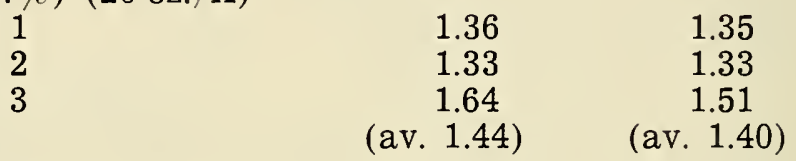

Guthion, ULV (98\%) (48 oz./A)

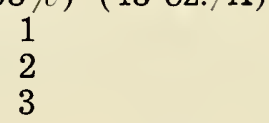

1.32

1.31

1.42

1.41

1.74

1.60

(av. 1.49)

(av. 1.44)

Malathion + Methoxychlor (3 qt./A)

$$
\begin{aligned}
& 1 \\
& 2 \\
& 3
\end{aligned}
$$

Malathion + Methoxychlor (2 qt./A)

$$
1
$$$$
1.06
$$

1.23

1.23

1.44

1.32

(av. 1.24)

(av. 1.19)

Guthion, ULV, (22\%) (48 oz./A)

$$
\begin{aligned}
& 1 \\
& 2 \\
& 3
\end{aligned}
$$

1.24

1.24

1.07

1.06

1.90

1.78

(av. 1.32)

(av. 1.31)

Malathion + Methoxychlor (2 qt./A)

$$
\begin{aligned}
& 1 \\
& 2 \\
& 3
\end{aligned}
$$


Malathion, LV, (20 oz./A)

1
2
3

Control

$\begin{array}{cc}1.67 & 1.60 \\ 1.20 & 1.18 \\ 1.23 & 1.22 \\ \text { (av. } 1.37) & \text { (av. } 1.33)\end{array}$

1
2
3
4

1.37

1.17

0.89

1.58

0.58

1.35

1.42

1.08

(av. 1.42)

(av. 0.99)

\section{B-WIDMYER FARM-Field 4}

Concentrate (undiluted) spray

Azinphosmethyl, EC. (20\%) (0.75) 50 oz.

$$
1
$$

2

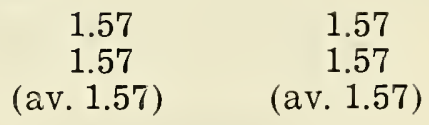

(av. 1.57)

Malathion, LV, (0.5) $10 \mathrm{oz}$.
1
2
1.00
1.00
0.96
0.96
(av. 0.98)
(av. 0.98)

SD-7438 EC. (25\%) (0.75) 50 oz.

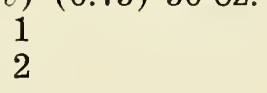

Malathion, EC. (0.75) $25 \mathrm{oz}$.

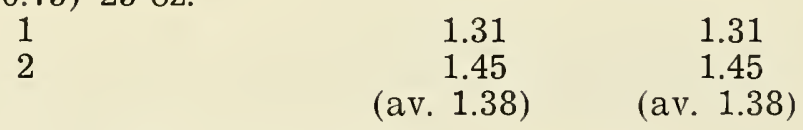

Azinphosmethyl, ULV. (22\%) (0.75) $50 \mathrm{oz}$.

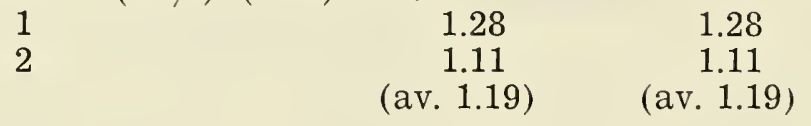

Control

\begin{tabular}{rrr}
1 & 1.57 & 0.89 \\
2 & 1.17 & 0.58 \\
3 & 1.58 & 1.42 \\
4 & 1.35 & 1.08 \\
& (av. 1.42) & (av. 0.99) \\
\hline
\end{tabular}


TABLE 6

Comparative effectiveness of uitra-low-volume foliar sprays applied with ground equipment to control the alfalfa weevil (1967).

Adult Weevils

Comp.

Treat- Effec-

ments $^{a}$ tiveness $^{b} \quad \%$ Kill
Weevil Larvae

Comp.

Treat- Effecments $^{a}$ tiveness $^{b} \%$ Kill

A-REEDSVILLE FARM (A1. S5) (Plots 4A x 2)

(Coll. 5-12-67)

$\begin{array}{rrrrrr}2 & \text { a } & 100.0 & 2 & \text { a } & 97.0 \\ 1 & \text { b } & 0.0 * & 1 & \text { b } & 0.0 \%\end{array}$

(Coll. 5-19-67)

$\begin{array}{rrrrrr}2 & \mathrm{a} & 71.0 & 2 & \mathrm{a} & 95.0 \\ 1 & \mathrm{a} & 0.0 & 1 & \mathrm{~b} & 0.0 * \%\end{array}$

aTable 6-A Treatments: (all LLV sprays applied in 1967 on Reedsville Farm with pressurized insecticide tank; 30 psi; applied $5-5-6 \overline{7}$ )

1. Untreated (geometric average adult weevil count 4.0; larval count 839.0)

2. Methyl parathion, EC (1.0) $(64 \mathrm{oz} / \mathrm{A})$

\section{B-REEDSVILLE (A1, S3 and 4) (Plots 4A $\times 2$ )}

(Coll. 5-12-67)

$\begin{array}{rrrrll}3 & \mathrm{a} & 82.0 & 2 & \mathrm{a} & 91.0 \\ 2 & \mathrm{a} & 75.0 & 3 & \mathrm{ab} & 79.0 \\ 1 & \mathrm{a} & 0.0 & 1 & \mathrm{~b} & 0.0^{* * * *}\end{array}$

(Coll. 5-19-67)

\begin{tabular}{llrrrr}
3 & $\mathrm{a}$ & 89.0 & 3 & $\mathrm{a}$ & 96.0 \\
2 & $\mathrm{ab}$ & 50.0 & 2 & $\mathrm{a}$ & 95.0 \\
1 & $\mathrm{~b}$ & 0.0 & 1 & $\mathrm{~b}$ & $0.0^{*}$ \\
\hline
\end{tabular}

-Table 6-B Treatments: (applicd 5-5-67)

1. Untreated (geometric average adult count 6.0; larval count 1147.0 )

2. Imidan, EC (1.0) $(64 \mathrm{oz} / \mathrm{A})$

3. Malathion, EC (1.0) (13 oz/A)

\section{C-REEDSVILLE (A3, S2) (Plots 2.25A x 2)}

(Coll. 5-26-67)

$\begin{array}{cccccc}3 & \mathrm{a} & 100.0 & 3 & \mathrm{a} & 95.0 \\ 2 & \mathrm{a} & 100.0 & 2 & \mathrm{a} & 93.0 \\ 1 & \mathrm{~b} & 0.0^{* *} & 1 & \mathrm{~b} & 0.0^{* *}\end{array}$

(Coll. 6-9-67)

$\begin{array}{rrrrll}2 & \mathrm{a} & 85.0 & 2 & \mathrm{a} & 92.0 \\ 3 & \mathrm{a} & 85.0 & 3 & \mathrm{ab} & 84.0 \\ 1 & \mathrm{a} & 0.0 & 1 & \mathrm{~b} & 0.0 \% * ;\end{array}$

'Table 6-C Treatments: (applied 5-19-6i)

1. Untreated (geometric average adult weevil count 6.0; larval count 1148.0)

2. Imidan, EC $(1.0)(64 \mathrm{oz} / \mathrm{A})$

3. Imidan, EC $(0.5)(32 \mathrm{oz} / \mathrm{A})$ 
D-REEDSVILLE FARM (Small Plots) (Plots 1/16 A x 2) (Coll. 5-12-67)

$\begin{array}{rrrrrr}2 & \mathrm{a} & 75.0 & 2 & \mathrm{a} & 7.0 \\ 1 & \mathrm{a} & 0.0 & 1 & \mathrm{a} & 0.0\end{array}$

(Coll. 5-19-67)

$\begin{array}{rrrrrr}2 & \text { a } & 0.0 & 2 & \text { a } & 52.0 \\ 1 & \text { a } & 0.0 & 1 & \text { a } & 0.0\end{array}$

"Table 6.1) Treatmens: (applied 52-67)

1. Entreated (geometric average adule weevil come 15.0); larval coumt 1147.0)

2. Baytex, LV $(0.5)(8-10 \% / / \Lambda)$

'Duncan's Multiple Range Test at level inclicated for Log $(X+1)$ of the data; antilog of data means -1 is presented as the geometric average count for 25 sweeps. Treatments sharing a letter in common do not differ in effectiveness.

"The aberrant data concerning adult weevil counts is mainly because of the scarcity of weevils at this time of the season. Negative control (more specimens in treated than in untreated plots) is acknowledged by $0.0 \%$ to indicate lack of control.

* 1.0 per cent level of significance.

* 5.0 per cent level of significance.

*** 10.0 per cent level of significance.

weevil control (adults and larvae) was accomplished one and two weeks post-treatment using Imidan (Table 6-C).

Economic control was not achieved with ULV sprays of Baytex at the rate of application used $(0.5 \mathrm{lb} / \mathrm{A})$ (Table 6-D) .

A 20-acre field was sprayed by aerial (helicopter) low-volume application using Imidan applied at two different rates $(0.5$ and $1.0 \mathrm{lb} / \mathrm{A})$. About two gallons of spray mixture per acre was applied. Economic adult and larval control resulted from this application one, two, and three weeks post-treatment (Table 7). The results obtained from 0.5 and $1.0 \mathrm{lb}$ rates were somewhat variable, but the material obviously was effective against the alfalfa weevil.

\section{Conclusions}

ULV and LV foliar spray treatment with malathion, azinphosmethyl, SD-7438. Imidan and methyl parathion correctly applied with ground equipment, and Imidan with aerial equipment, are effective against the alfalfa weevil.

Hydrocarbons (Sun Oil 7EL and TSEL) applied as ULV treatments on alfalfa stubble (first cutting) are as effective as either malathion or azinphosmethyl in killing adult and larval stages of the alfalfa weevil.

ULV concentrate sprays of Sun Oil 91 (naphthenic) applied in mid-November and 91EL applied in late March are economically effective in reducing alfalfa weevil populations. 

foliage with aerial equipment to control the alfalfa weevil (196\%).

\begin{tabular}{|c|c|c|c|c|c|}
\hline \multicolumn{3}{|c|}{ Adult Weevils } & \multicolumn{3}{|c|}{ Weevil Larvae } \\
\hline $\begin{array}{l}\text { Treat- } \\
\text { ments }^{a}\end{array}$ & $\begin{array}{c}\text { Comp. } \\
\text { Effec- } \\
\text { tiveness }^{b}\end{array}$ & $\%$ Kill & $\begin{array}{l}\text { Treat- } \\
\text { ments }\end{array}$ & $\begin{array}{c}\text { Comp. } \\
\text { Effec- }^{-} \\
\text {tiveness }^{b}\end{array}$ & $\%$ Kill \\
\hline \multicolumn{6}{|c|}{$\begin{array}{c}\text { DEMORY FARM (Field 1) } \\
(\text { Coll. 5-3-6y) }\end{array}$} \\
\hline 2 & a & $100 \cdot 0$ & 2 & a & 81.0 \\
\hline 1 & $\bar{a}$ & 0.0 & 3 & $\mathrm{a}$ & 51.0 \\
\hline 3 & $a$ & $0.0^{\mathrm{c}}$ & 1 & $\mathrm{a}$ & 0.0 \\
\hline \multicolumn{6}{|c|}{ (Coll. 5-10-67) } \\
\hline 3 & a & 100.0 & 3 & a & 98.0 \\
\hline 2 & a & 100.0 & 2 & $a b$ & 82.0 \\
\hline 1 & $\mathrm{~b}$ & $0.0 *$ & 1 & $\mathrm{~b}$ & $0.0 * *$ \\
\hline \multicolumn{6}{|c|}{ (Coll. 5-16-67) } \\
\hline 3 & a & 100.0 & 2 & a & 99.0 \\
\hline 2 & $a b$ & 66.0 & 3 & $\mathrm{a}$ & 40.0 \\
\hline 1 & $\mathrm{~b}$ & $0.0 *$ & 1 & a & 0.0 \\
\hline
\end{tabular}

aTreatments: (helicopter sprayer (Bell 47--D-1), 35-50 mph, 3-6 ft. above ground, 50 ft. swath; applied 4-27-67)

1. Untreated (geometric average adult weevil count 2.0 ; larval count 42.0)

2. Imidan, $3 \mathrm{E}(0.5)$ (1 gal $\mathrm{mix} / \mathrm{A}$ )

3. Imidan, 3E (1.0) (2 gal mix/A)

${ }^{b}$ Duncan's Multiple Range Test at level indicated for $\log (\mathrm{N}+1)$ of the data; antilog of data means -1 is presented as the geometric average count for 25 swceps. Treatments sharing a letter in conmon do not differ in effectiveness.

${ }^{c}$ The aberrant data concerning adult weevil counts is mainly because of the scarcity of weevils at this time of the season. Negative control (more specimens in treated than in untreated plots) is acknowledged by $0.0 \%$ to indicate lack of control.

* 1.0 per cent level of significance.

* 5.0 per cent level of significance.

\section{Literature Cited}

Adair, H. M.. R. T. Kincade, M. L. Laster, and J. R. Brazell. 1967. Low-volume aerial spraying of several insecticides for cotton insect control. J. Econ. Entomol. 65 (4): 1121-27.

Adkisson, P L 1966. Low-volume sprays of guthion and malathion for reducing diapausing boll weevil Anthonomis grandis poptulations. Tex. Agr. Exp. Sta. Pro. gress Rep. PR-2405.

Bemett, S. and H. Lutuell. 1965. Alfalfa weevil larval control with low-rolume application of technical malathion. Tenn. Farm Home Sci. Progress Rep. 56:22-23. 
Burgress, E. D. 1965. Control of boll weevil with Technical malathion applied by aircraft. J. Econ. Entomol. 58 (3): 414-15.

Burt, E. C., D. B. Smith, and E. P. Lloydl. 1966. A rotary disc device for applying ULV (undiluted) pesticides with ground equipment. J. Econ. Entomol. 59 (6): 1487-89.

Cleveland T. C., W. P. Scott, T. B. Davich, and C. R. Parencia, Jr. 1966. Control of the boll weevil on cotton with ultra-low-volume (undiluted) technical malathion. J. Econ. Entomol. 59 (4): 973-76.

Harrell, E. A., W. W. Hare, and J. R. Young. 1966. Ground equipment for applying low-volume insecticides to sweet corn. J. Econ. Entomol. 59 (2): 487-89.

Harrell, E. A., J. R. Young, M. C. Bowman, and W. W. Hare. 1967. Insect control and residues in sweet corn using ground cquipment for treating with low-volume formulations. J. Econ. Entomol. 60 (4): 988-91.

Harrell, E. A. and D. B. Leuck. 1967. Ultra-low-volume ground equipment for applying insecticides to soybeans. J. Econ. Entomol. 60 (4): 1164-65.

MacCuaig, R. D. 1966. Toxicity of ultra-low-volume sprays. J. Econ. Entomol. 59 (4): 1009-10.

Messenger, K. 1964. Low-volume aerial spraying. Agr. Chem. 19 (9): 61-4.

Messenger, K. 1965. Liquid concentrates for insect control. Proc. Beltwide Cotton Production Mechanization Conf. (1965).

Niemczyk, H. D., J. E. Henry, and W. W. Roberts. 1967. Aerial applications of malathion LV concentratc for control of alfalfa weevil in Ohio. J. Econ. Entomol. 60 (4): $1000-2$.

Pass, B. C. and F. W. Knapp. 1966. Aerial application of insecticides for control of alfalfa weevil. J. Econ. Entomol. 59 (3): 648-50.

Skoog, F. E., F. T. Cowan, and K. Messenger. 1965. Ultra-low-volume aerial spraying of dicldrin and malathion for rangeland grasshopper control. J. Econ. Entomol. 58 (3): 559-65.

Thomas, C. A. 1966. Low-volume concentrated sprays applied by ground equipment for control of the boll weevil Anthonomis grandis. J. Econ. Entomol. 59 (1): 114-16.

Wilson, M. C., R. F. Ruppel, and R. E. Treece. 1965. Low-volume concentrate sprays applied by aircraft for control of cereal leaf beetle. J. Econ. Entomol. 58 (1): 11-14. 


